\title{
Primary Leiomyosarcoma of the Inferior Vena Cava: is vascular reconstruction always necessary?
}

Nizar Kardoun ${ }^{1}$, Ahmed

Bouzid $_{a}$ hmed ${ }^{1}$, AymanTrigui $^{1}$, HaithamRejab $^{1}$, MohammedBenAmar $^{1}$, andRafikMzali $^{1}$

${ }^{1}$ Habib Bourguiba Hospital

August 20, 2021

\begin{abstract}
Primary leimyosarcoma of the inferior vena cava is considered as rare vascular retroperitoneal sarcoma. Although radical resection with free margin is necessary, vascular strategies are very challenging.
\end{abstract}

Title: Primary Leiomyosarcoma of the Inferior Vena Cava: is vascular reconstruction always necessary?

Authors: Nizar Kardoun ${ }^{1}$, Ahmed Bouzid ${ }^{1, \&}$, Ayman Trigui ${ }^{1}$, Haithem Rejab ${ }^{1}$, BenAmar Mohamed ${ }^{1}$, Mzali Rafik ${ }^{1}$

Affiliations: ${ }^{1}$ General surgery Department, Habib Bourguiba Hospital, Sfax, TN 3089

Corresponding Author: \& General Surgery Department Habib Bourguiba Hospital University of Sfax Route ElAin 0.5km Sfax, TN 3021, Phone Number: 20679500

Abstract: Primary leimyosarcoma of the inferior vena cava is considered as rare vascular retroperitoneal sarcoma. Although radical resection with free margin is necessary, vascular strategies are very challenging.

KEYWORDS: Primary leimyosarcoma, inferior vena cava, vascular reconstruction

Key Clinical Message: The study of preoperative imaging is essential for a better surgical strategy. The vascular reconstruction depends essentially on the location and the size of the tumor best evaluated intraoperatively.

A 65-year-old woman without past medical history presented with intermittent right upper quadrant abdominal pain. The physical exam revealed no characteristic features. Laboratory exam were normal.

Abdominal CT revealed a $6.5^{*} 5 \mathrm{~cm}$ heterogeneous non calcified soft-tissue mass. This mass was invading the inferior vena cava (IVC) and abutting to the right renal vein (figure 1). No focal other mass was observed in the abdominal cavity. Regarding the high risk of malignancy, an exploratory laparotomy was performed. During laparotomy, the tumor was adherent to the right lateral side of IVC without evidence of endoluminal development (figure 2).

A radical en bloc resection of the tumor was performed with lateral resection of the vena cava with primary closure.

Pathology confirmed R0 resection of a grade 1 primary leiomyosarcoma of the inferior vena cave (PLV). Immunohistochemically, the tumor cells were positive for PS-100 and desmin and negative for CK and c-kit.

PLV a rare retroperitoneal sarcomas originating from the smooth muscle cells of the media with intra or extra-luminal growth ${ }^{1}$. Although vascular resection with free margin is necessary, vascular reconstruction 
without graft interposition is not always necessary. The vascular strategies depends essentially on the location and the size of the tumor ${ }^{2}$.

Author contribution statement: Nizar Kardoun: writing -review and editing (equal); Ahmed Bouzid: Writing - review and editing (equal); Ayman Trigui: review and editing; Haithem Rejab: Writing - review and editing (equal); BenAmar Mohamed: Conceptualization, Mzali Rafik: Validation- Supervision

References:

1. Dew J, Hansen K, Hammon J, McCoy T, Levine EA, Shen P. Leiomyosarcoma of the Inferior Vena Cava: Surgical Management and Clinical Results.Am Surg . 2005;71(6):497-501. doi:10.1177/000313480507100609

2. Ruiz CS, Kalbaugh CA, Browder SE, et al. Operative strategies for inferior vena cava repair in oncologic surgery. J Vasc Surg Venous Lymphat Disord . 2020;8(3):396-404. doi:10.1016/j.jvsv.2019.09.012

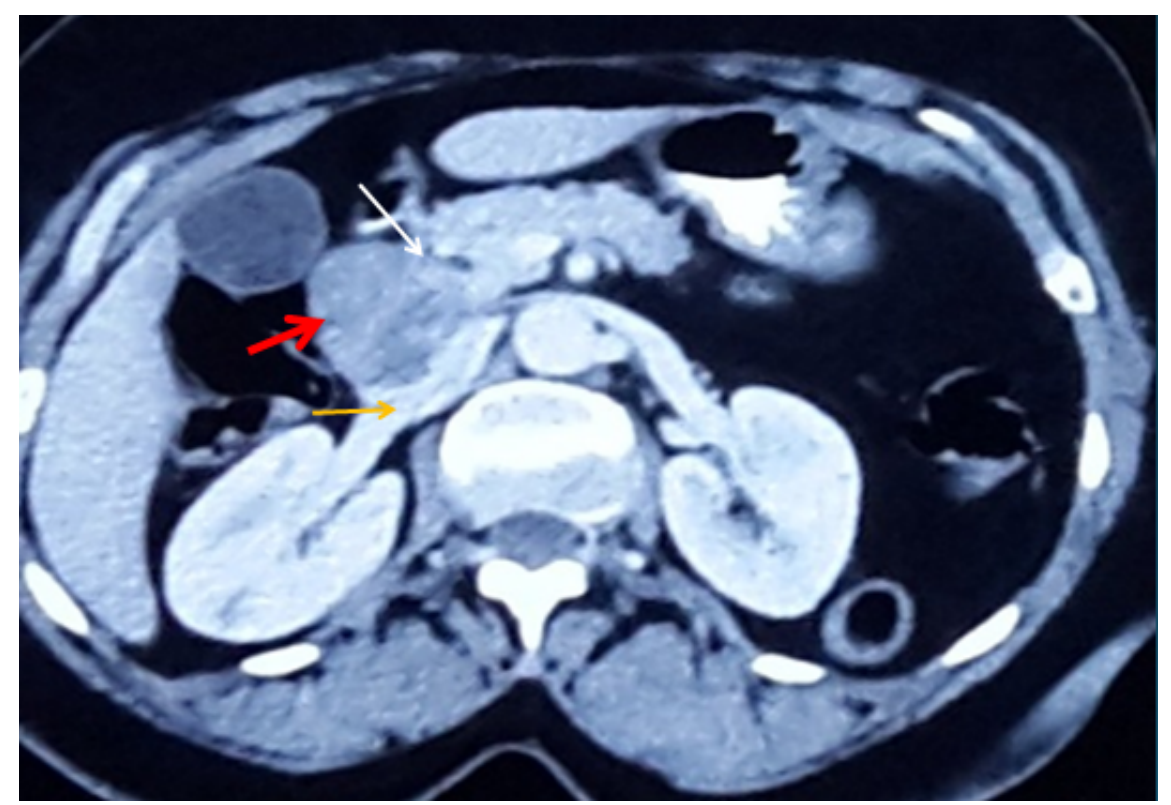




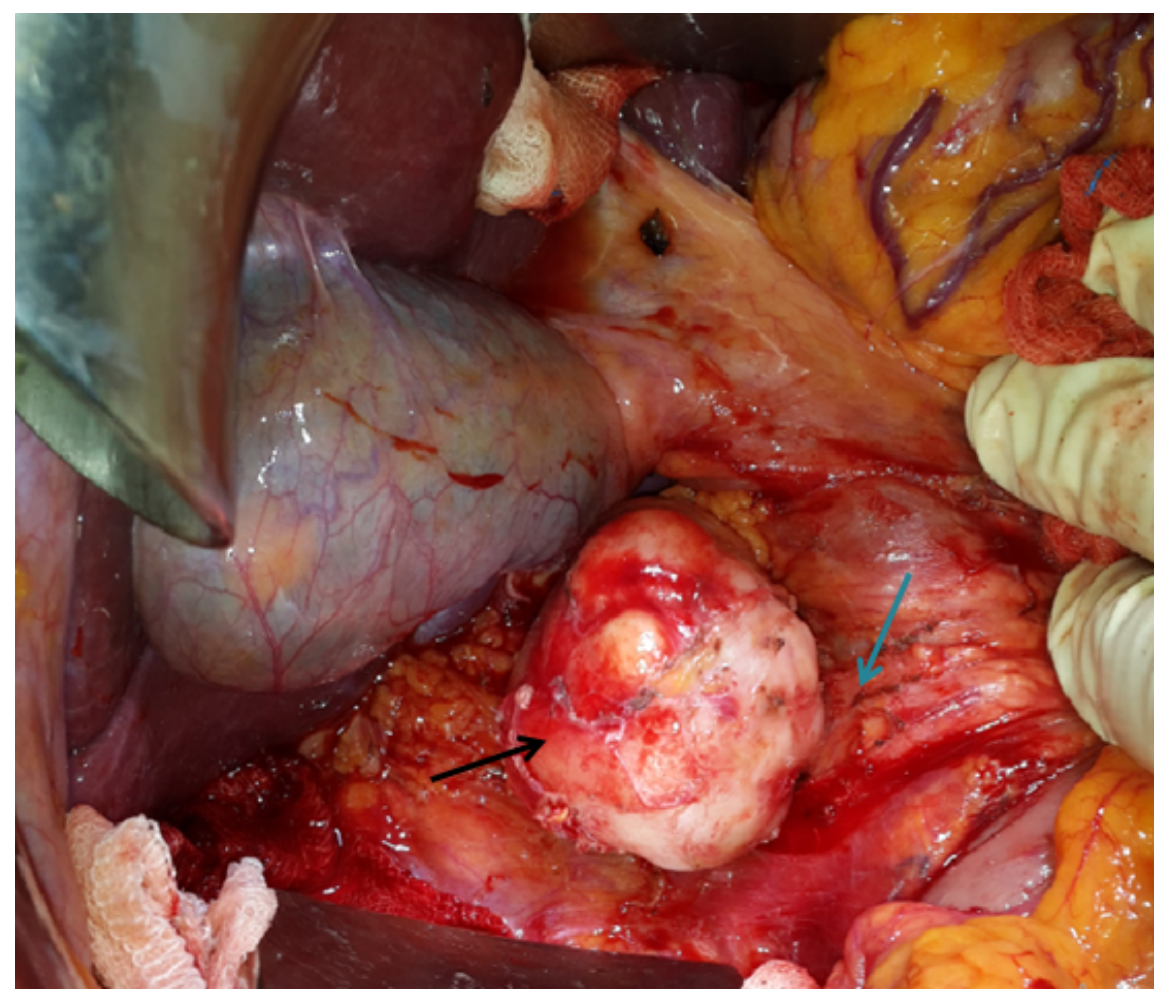

